Research Paper

\title{
Development of a New Classification Method for Penile Squamous Cell Carcinoma Based on Lymph Node Density and Standard Pathological Risk Factors: The ND Staging System
}

Zai-Shang Li 1,2,3†, Kai Yao 1,2,3†, Peng Chen 4 , Bin Wang 5 , Oi-Wu Mi 6 , Jie-Ping Chen 1,2,3, Yong-Hong Li 1,23

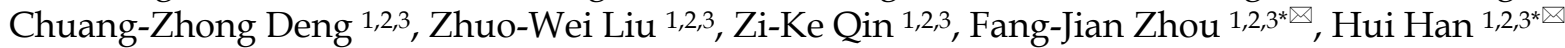

1. Department of Urology, Sun Yat-sen University Cancer Center, Guangzhou, P. R. China

2. State Key Laboratory of Oncology in Southern China, Guangzhou, P. R. China

3. Collaborative Innovation Center of Cancer Medicine, Guangzhou, P. R. China

4. Department of Urology, Affiliated Tumor Hospital of Xinjiang Medical University, Urumchi, P. R. China

5. Department of Urology, Cancer Center of Guangzhou Medical University, Guangzhou, P. R. China

6. Department of Urology, Dong Guan People's Hospital, Guang Dong, P. R. China

$\dagger$ These authors contributed equally to this research.

* The last two authors equally contributed to this work for correspondence.

$\square$ Corresponding authors: Hui Han M.D., Ph.D., Professor, Department of Urology, Sun Yat-sen University Cancer Center, Guangzhou 510060, P.R. China Tel: 86-20-87343656; Fax: 86-20-87343656, e-Mail: hanhui@sysucc.org.cn. Fang-Jian Zhou, M.D., Ph.D., Professor, Department of Urology, Sun Yat-sen University Cancer Center, Guangzhou 510060, P.R. China Tel: 86-20-87343952; Fax: 86-20-87343952, e-Mail: zhoufj@sysucc.org.cn.

() Ivyspring International Publisher. Reproduction is permitted for personal, noncommercial use, provided that the article is in whole, unmodified, and properly cited. See http://ivyspring.com/terms for terms and conditions.

Received: 2015.07.07; Accepted: 2015.11.13; Published: 2016.01.05

\begin{abstract}
Object: In this study, we evaluated the role of lymph node density (LND) and validated whether LND increases the accuracy of survival prediction when combined with the American Joint Committee on Cancer (AJCC) pathological node $(N)$ staging system for penile cancer ( $7^{\text {th }}$ edition).

Methods: A total of 270 Chinese penile cancer patients treated between March 1999 and October 2014 were retrospectively analyzed. LND was analyzed as a trichotomous variable for the prediction of DSS in this cohort. We developed a new prediction model, which we refer to as the ND staging system, that is based on LND and pathological $N$ staging. The predictive accuracy of this model was further assessed using the concordance index.

Results: LND was correlated with the laterality of lymph node metastasis, extranodal extension, pelvic lymph node metastases, and pathologic tumor $(T)$ and $N$ stages $(P<0.05)$. In separate multivariate Cox regression models, the LND (hazard ratio [HR], 1.966, 95\% confidence interval [Cl], $(1.112-3.473, P=0.020)$ yielded independent effects on the outcome. According to the LND classification, the 3-year disease-specific survival (DSS) rates for patients with LNDs $<7.0 \%, 7.0$ to

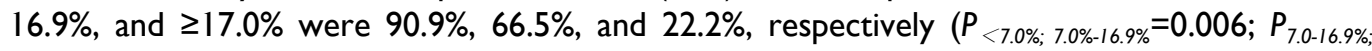
$\geq 17.0 \%=0.001$ ). The corresponding rates were $95.7 \%, 76.7 \%$, and $28.1 \%$ for the ND1, ND2, and ND3 patients, respectively $\left(P_{\mathrm{NDI}-\mathrm{ND2}}=0.047 ; P_{\mathrm{ND2}-\mathrm{ND} 3}<0.001\right)$. The indexes indicated that the accuracy of the pathological ND category that incorporated LND was significantly increased.

Conclusion: LND was associated with some prognosticators and is thus a prognostic factor. The ND staging system that incorporates the LND better reflects the prognoses of penile cancer patients.
\end{abstract}

Key words: penile neoplasm, staging system, lymph node metastasis, lymph node density, prognosis 


\section{Introduction}

The inguinal lymph node metastasis (LNM) status is one of the most important prognostic factors in patients with penile squamous cell carcinoma (SCC) [1-3]. However, as in other tumors, the number of LNMs removed is affected by various factors that bias survival analyses, such as the method of lymph node resection, the pathologist's assessment, and individual physiological variations ${ }^{[4-6]}$.

The lymph node density (LND) is defined as the total number of LNMs that are affected by tumors divided by the total number of lymph nodes (LNs) removed [7-9]. This predictive factor simultaneously reflects the extent of nodal dissection and nodal disease burden. The LND is used as a prognostic factor for other solid tumors, such as those of bladder cancer $[4]$, colorectal cancer [10], and gastric cancer [11]. The significance of the LND for penile cancer was first reported in 2009 by Pettaway et al. [7]. The 2014 European Association of Urology (EAU) first recommended the use of LND for the prediction of the prognoses of patients with penile cancer [2]; however, few studies have examined the optimal LND cutoff that should be used to predict the survival of patients with SCC. The goal of our study was to evaluate the prognostic value of LND in the prediction of DSS in a large group of node-positive $(\mathrm{N}+)$ penile cancer patients undergoing bilateral inguinal lymphadenectomy (ILND).

\section{Materials and methods}

\section{Patient Population}

The clinical and pathological data of 325 patients with penile carcinomas who were treated at the Sun Yat-Sen University Cancer Center from March 1999 to October 2014 were reviewed. The eligibility criteria were histologically confirmed penile SCC and bilateral ILND. The ages at surgery ranged from 18 to 85 years. Cases with the following criteria were excluded from the study: neoadjuvant chemotherapy/radiotherapy, clinical evidence of distant metastasis, resection of the primary lesion only, and loss to follow-up. Only 270 patients met the study criteria.

\section{Treatment and Study Assessments}

According to the guidelines of the EAU, ILND is recommended for all penile SCC patients. All of the ILNDs were performed by the same surgeons. The boundaries and technologies of ILND and the treatment criteria were previously described in detail $[8,12]$. All of the patients received follow-ups every 3 months for the first 2 years following surgery, every 6 months in the 3rd and 4th years, and once yearly thereafter. The deadline for follow-up was March 2015.

\section{Statistical Analyses}

LND was calculated using the following formula: the number of positive LNs divided by the total number of removed LNs multiplied by $100 \%$. For fixed LNM patients with a number of harvested LNs that was less than the mean, the number of LNs was defined as the average of the harvested nodes, and the number of positive LNs was defined as the total number of LNs minus the number of negative LNs.

This interval-scaled continuous variable was trisected. Categorical variables were compared between groups using the $\chi^{2}$ test. We did not evaluate the role of adjuvant therapies using multivariate analyses because such therapies were not routinely administered to all of the enrolled patients. The Kaplan-Meier method was used to determine the DSS rates. Several risk factors for SCC that were identified by the guidelines were added to the regression. Due to the collinear association of the LND with the number of LNMs $(R=0.91)$, separate regression models were developed. Because the extranodal extension (ENE) and pelvic LNM are included in the $7^{\text {th }} \mathrm{N}$ staging system, only additional factors were added to the regression. Univariate and multivariate Cox regression models were fitted to assess the predictors of DSS. The LR, AIC, and c-index were investigated to evaluate the accuracies of the models. Bootstrap-corrected c-indexes were used for internal validation to better gauge the expected future predictive accuracy (500 samplings). All of the statistical analyses were performed with R2.11.1 (http://www.r-project.org), and $P<0.05$ indicated statistical significance.

\section{Results}

\section{Patient Characteristics}

Among the 270 enrolled patients, the mean number of LNs was 24 (median 23, range 8 to 63), and 144 patients had LNM. Of the latter patients, 55 patients died of penile cancer at a mean of 21.6 months (median 11.9 months, range 1.2 to 101.8 months). There are 23 patients with fused LNM, of which $8(5.6 \%, 11 / 144)$ patients with a number of harvested LNs that was less than the mean. For the latter, the mean number of harvested LNs was 15 (median 16, range 12 to 22). The clinical and pathological characteristics of the patients are presented in Table 1.

\section{LND and Clinicopathological Characteristics}

The LNDs were divided into the following three percentiles by trisection: $<7.0 \%, 7.0 \%$ to $16.9 \%$, and $\geq 17.0 \%$. Table 2 reveals that the LNM laterality, ENE, pelvic LNM, and $\mathrm{T}$ and $\mathrm{N}$ stages can be different according to LNDs $(P<0.05)$. Moreover, $91.1 \%(51 / 56)$ of 
the ENEs, $94.7 \%(54 / 57)$ of the bilateral LNMs, and 95.7\% (22/23) of the pelvic LNMs were characterized by LNDs $\geq 7.0 \%$. No significant differences were noted between the groups with respect to smoking history $(P=0.085)$, phimosis $(P=0.778)$, or $\mathrm{G}$ stage $(P=0.273)$.

\section{Survival Predictor}

The univariate analysis factors that were significantly associated with decreased DSS included pathological LNM laterality, T stage, N stage, G, and LND. Smoking history and phimosis were not significantly associated with increased risks of death from the disease. The results of the multivariate analyses of the multiple prognostic variables for DSS according to nodal status are summarized in Table 3. After controlling for the other pathological variables, only LND continued to exert an independent influence on DSS in the $\mathrm{N}+$ patients.

The three-year DSS rates were $92.6 \%, 66.5 \%$, and $27.5 \%$ for the N1, N2, and N3 patients, respectively, based on the $7^{\text {th }}$ edition $\mathrm{N}$ (node) staging system $\left(P_{\mathrm{N} 1-\mathrm{N} 2}=0.088, P_{\mathrm{N} 2-\mathrm{N} 3}<0.001, P<0.001\right.$, respectively; Fig. 1A). The three-year DSS rates for the corresponding LND groups were $90.9 \%, 66.5 \%$, and $22.2 \%\left(P_{<7.0 \%}\right.$; 7.0-16.9\% $=0.006, P_{7.0 \%-16.9 \% ; \geq 17.0 \%}=0.001, P<0.001$; Fig. $\left.1 B\right)$. After pathological review, the LN statuses were reassessed according to the $7^{\text {th }}$ edition of the American Joint Committee on Cancer (AJCC) tumor, node, and metastasis (TNM) staging system and the modified pathological $\mathrm{N}$ staging system (Table 4). The corresponding DSS rates were $95.7 \%, 76.7 \%$, and $28.1 \%$ for the ND1, ND2, and ND3 patients, respectively $\left(P_{N D 1-N D 2}=0.047 ; P_{N D 2-N D 3}<0.001, P<0.001\right.$, respectively, Fig. 1C). The addition of LND significantly increased the predictive accuracy of the basic model (Table 5). The bootstrap-corrected c-index of the modified $\mathrm{N}$ stage categories was 0.764 , which was inferior to that of the $7^{\text {th }}$ pathological $\mathrm{N}$ staging system $(P<0.001)$.

Table 1. Clinical and pathological characteristics of 144 patients

\begin{tabular}{|c|c|c|c|c|}
\hline Variable & $\mathrm{N}(\%)$ & 1-year DSS (95\% CI) & 3-year DSS (95\% CI) & $P$-value \\
\hline Age at surgery, year, median (range) & $50(24-82)$ & & & - \\
\hline BMI, kg/m², median (range) & $22.4(15.4-36.7)$ & & & - \\
\hline Smoking History & & & & 0.053 \\
\hline Yes & $75(52.1)$ & $78.4(62.8-88.6)$ & $63.9(49.4-78.4)$ & \\
\hline No & $69(47.9)$ & $63.1(51.1-75.1)$ & $44.0(29.1-58.9)$ & \\
\hline Phimosis & & & & 0.173 \\
\hline Yes & $89(61.8)$ & $70.4(60.4-80.4)$ & $62.8(51.4-74.2)$ & \\
\hline No & $55(38.2)$ & $71.9(59.2-84.6)$ & $34.0(13.2-54.8)$ & \\
\hline Number of LNs, $n$, median (range) & $24(8-63)$ & & & 0.547 \\
\hline$<24$ & $61(42.4)$ & $75.2(63.4-87.0)$ & $58.1(42.6-73.6)$ & \\
\hline$\geq 24$ & $83(57.6)$ & $67.6(57.0-78.2)$ & $51.0(36.5-65.5)$ & \\
\hline LNM laterality (n) & & & & 0.001 \\
\hline Unilateral & $87(60.4)$ & $78.4(64.7-92.1)$ & $68.4(56.1-80.7)$ & \\
\hline Bilateral & $57(39.6)$ & $58.7(44.8-72.6)$ & $31.3(14.8-47.8)$ & \\
\hline $\operatorname{ENE}(\mathrm{n})$ & & & & $<0.001$ \\
\hline Yes & $56(38.9)$ & $55.2(41.3-69.1)$ & $27.3(11.4-43.2)$ & \\
\hline No & $88(61.1)$ & $80.4(71.4-89.4)$ & $71.2(59.4-83.0)$ & \\
\hline Pelvic LNM (n) & & & & 0.034 \\
\hline Yes & $23(16.0)$ & $52.6(30.6-74.6)$ & $26.3(11.7-63.4)$ & \\
\hline No & $121(84.0)$ & $74.3(65.9-82.7)$ & $57.1(45.9-68.3)$ & \\
\hline Number of LNMs (n) & & & & $<0.001$ \\
\hline 1 & $36(25.0)$ & $89.8(78.8-100.0)$ & $89.8(78.8-100.0)$ & \\
\hline 2 & $42(29.2)$ & $76.5(62.0-91.0)$ & $57.7(35.6-79.8)$ & \\
\hline$\geq 3$ & $66(45.8)$ & $56.7(44.2-69.2)$ & $28.6(13.9-43.3)$ & \\
\hline $\mathrm{T}(\mathrm{n})$ & & & & $<0.001$ \\
\hline$\leq \mathrm{T} 1$ & $18(12.5)$ & $88.1(72.6-100.0)$ & $70.5(67.2-73.8)$ & \\
\hline $\mathrm{T} 2$ & $95(68.5)$ & $76.1(66.9-85.3)$ & $59.0(46.1-71.9)$ & \\
\hline$\geq \mathrm{T} 3$ & $31(16.2)$ & $45.0(26.6-63.4)$ & $27.4(8.0-46.8)$ & \\
\hline $\mathrm{G}(\mathrm{n})$ & & & & 0.070 \\
\hline 1 & $68(47.2)$ & $77.9(67.1-88.7)$ & $55.3(38.8-71.8)$ & \\
\hline 2 & $54(37.5)$ & $69.7(56.6-82.8)$ & $58.6(42.1-75.1)$ & \\
\hline 3 & $22(15.3)$ & $51.0(29.0-73.0)$ & $36.4(13.3-59.5)$ & \\
\hline $7^{\text {th }} \mathrm{N}(\mathrm{n})$ & & & & $<0.001$ \\
\hline N1 & $28(19.4)$ & $92.6(82.6-100.0)$ & $92.6(82.6-100.0)$ & \\
\hline N2 & $50(34.7)$ & $80.4(67.9-92.9)$ & $66.5(48.5-84.5)$ & \\
\hline N3 & $66(45.8)$ & $53.3(40.6-66.0)$ & $27.5(13.0-42.0)$ & \\
\hline LND (n) & & & & $<0.001$ \\
\hline$<7.0$ & $42(29.2)$ & $90.9(80.9-100.0)$ & $90.9(80.9-100.0)$ & \\
\hline $7.0-16.9 \%$ & $49(34.0)$ & $72.0(58.3-85.7)$ & $66.5(50.0-83.0)$ & \\
\hline$\geq 17.0 \%$ & $53(36.8)$ & $53.7(39.8-67.6)$ & $22.2(8.3-36.1)$ & \\
\hline
\end{tabular}


Table 2. The relationship between patient clinical-pathological characteristics and LND

\begin{tabular}{|c|c|c|c|c|}
\hline Variable & $<7.0 \%$ & $7.0-16.9 \%$ & $\geq 17.0 \%$ & $P$-value \\
\hline Smoking History & & & & 0.085 \\
\hline Yes & $16(11.1)$ & $27(18.8)$ & $32(22.2)$ & \\
\hline No & $26(18.1)$ & $22(15.3)$ & $21(14.6)$ & \\
\hline Phimosis & & & & 0.778 \\
\hline Yes & $26(18.1)$ & $32(22.2)$ & $31(21.5)$ & \\
\hline No & $16(11.1)$ & $17(11.8)$ & $22(15.3)$ & \\
\hline LNM laterality & & & & $<0.001$ \\
\hline Unilateral & $39(27.1)$ & $26(18.1)$ & $22(15.3)$ & \\
\hline Bilateral & $3(2.1)$ & $23(16.0)$ & $31(21.5)$ & \\
\hline ENE & & & & $<0.001$ \\
\hline Yes & $5(3.5)$ & $11(7.6)$ & $40(27.8)$ & \\
\hline No & $37(25.7)$ & $38(26.4)$ & $13(9.0)$ & \\
\hline Pelvic LNM & & & & 0.006 \\
\hline Yes & $1(0.7)$ & $8(5.6)$ & $14(9.7)$ & \\
\hline No & $41(28.5)$ & $41(28.5)$ & $39(27.1)$ & \\
\hline \multicolumn{5}{|l|}{$\mathrm{T}(\%)$} \\
\hline$\leq \mathrm{T} 1$ & $8(5.6)$ & $6(4.2)$ & $4(2.8)$ & 0.004 \\
\hline $\mathrm{T} 2$ & $31(21.5)$ & $35(24.3)$ & $29(20.1)$ & \\
\hline$\geq \mathrm{T} 3$ & $3(2.1)$ & $8(5.6)$ & $20(13.9)$ & \\
\hline $\mathrm{G}(\%)$ & & & & 0.273 \\
\hline G1 & $23(16.0)$ & $26(18.1)$ & $19(13.2)$ & \\
\hline G2 & $15(10.4)$ & $16(11.1)$ & $23(16.0)$ & \\
\hline G3 & $4(2.8)$ & $7(4.9)$ & $11(7.6)$ & \\
\hline $\mathrm{N}(\%)$ & & & & $<0.001$ \\
\hline N1 & $24(16.7)$ & $4(2.8)$ & 0 & \\
\hline N2 & $13(9.0)$ & $29(20.1)$ & $8(5.6)$ & \\
\hline N3 & $5(3.5)$ & $16(11.1)$ & $45(31.3)$ & \\
\hline
\end{tabular}

Table 3. Multivariate Cox regression analyses for DSS

\begin{tabular}{|c|c|c|c|}
\hline \multirow[t]{2}{*}{ Variable } & \multicolumn{3}{|c|}{ Multivariate analysis } \\
\hline & HR & CI $(95 \%)$ & $P$-value \\
\hline \multicolumn{4}{|l|}{ Smoking History } \\
\hline Yes VS No & 1.457 & $0.766-2.769$ & 0.251 \\
\hline \multicolumn{4}{|l|}{ Phimosis } \\
\hline Yes VS No & 0.568 & $0.297-1.084$ & 0.086 \\
\hline \multicolumn{4}{|l|}{ LNM laterality } \\
\hline \multicolumn{4}{|l|}{$\mathrm{T}$} \\
\hline$\leq \mathrm{T} 1$ VS T2 VS $\geq \mathrm{T} 3$ & 1.607 & $0.923-2.798$ & 0.093 \\
\hline \multicolumn{4}{|l|}{$\mathrm{N}$} \\
\hline N1 VS N2 VS N3 & 1.729 & $0.942-3.174$ & 0.077 \\
\hline \multicolumn{4}{|l|}{ G } \\
\hline G1 VS G2 VS G3 & 1.407 & $0.948-2.088$ & 0.091 \\
\hline \multicolumn{4}{|l|}{ LND } \\
\hline D1 VS D2 VS D3 & 1.966 & $1.112-3.473$ & 0.020 \\
\hline
\end{tabular}

Table 4. AJCC N staging system (7th edition) and the ND staging system

\footnotetext{
Stage AJCC N staging system (7th $\quad$ Modified N staging system edition)

N0 No regional lymph node me- No regional lymph node metastasis tastasis

Nx Regional lymph nodes cannot Regional lymph nodes cannot be be assessed assessed

N1 Metastasis in a single inguinal Metastasis in a single inguinal lymph lymph node node or LND $<7.0 \%$

N2 Metastasis in multiple or bilat- Metastasis in multiple or bilateral eral inguinal lymph nodes inguinal lymph nodes or $\mathrm{LND}=7.0-16.9 \%$

N3 Unilateral or bilateral ENE of Unilateral or bilateral ENE of LNM or LNM or pelvic lymph node(s) pelvic lymph node(s) or LND $\geq 17 \%$

ENE: extranodal extension, LNM: lymph node metastasis, LND=lymph node density
}

Table 5. Predictive accuracy of models: $N$ staging system ( $7^{\text {th }}$ edition) and ND staging system

\begin{tabular}{lcccccc}
\hline Models & HR * & CI * & LR & AIC & c-index & $\begin{array}{l}\text { Bootstrap } \\
\text { C-index }\end{array}$ \\
\hline $\begin{array}{l}\text { 7th N staging } \\
\begin{array}{l}\text { system } \\
\text { ND staging } \\
\text { system }\end{array}\end{array}$ & 4.040 & $2.250-7.256$ & 28.418 & 167.100 & 0.737 & 0.736 \\
\hline *Logistic, AIC=Akaike information criterion, LR=likelihood ratio, & & & & \\
\hline
\end{tabular}
c-index $=$ concordance index
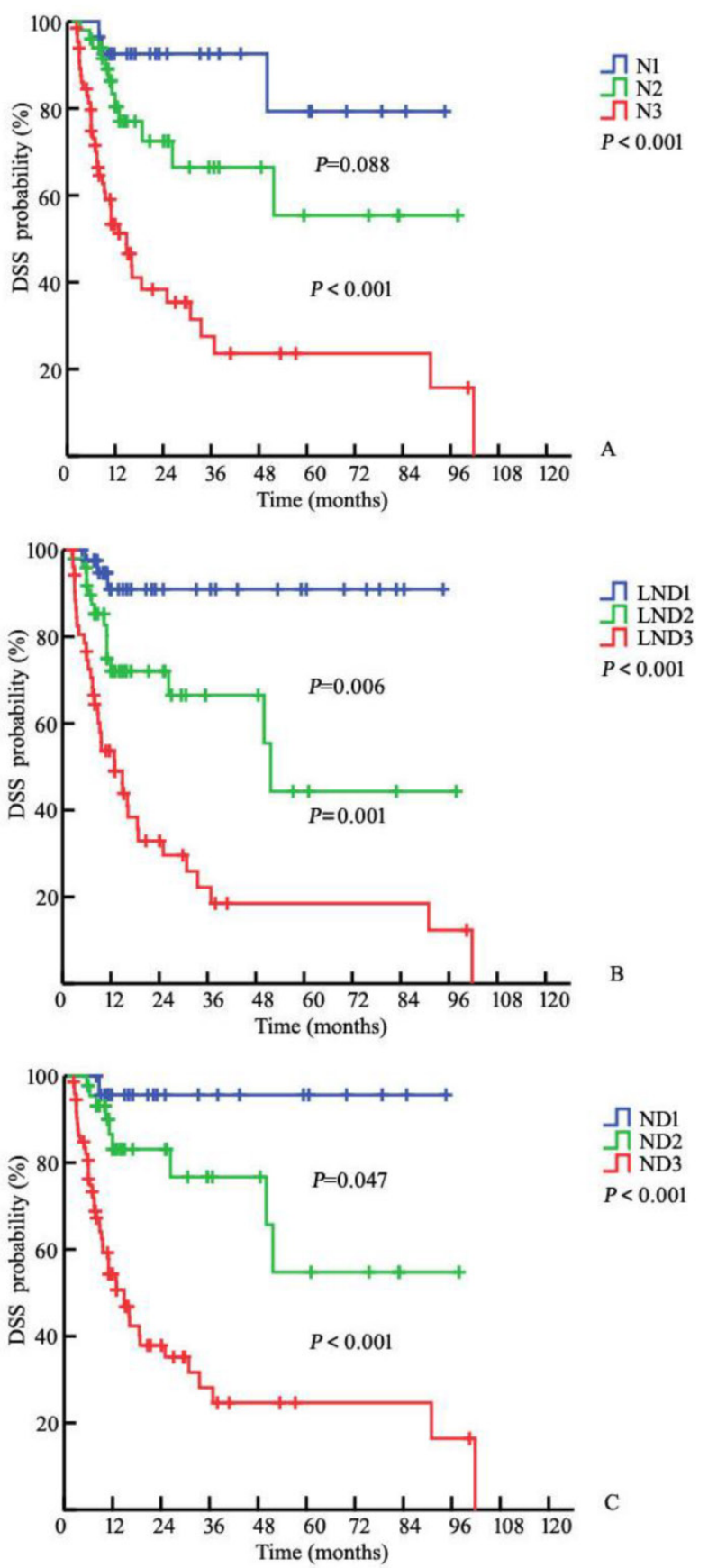

Figure 1. Kaplan-Meier estimates for DSS stratified by the $7^{\text {th }} N(P<$ $0.001)(A), L N D(P<0.001)(B)$, and N+LND (ND) $(C)$ staging systems 


\section{Discussion}

We examined the prognostic significance of LND for the prediction of DSS in $\mathrm{N}+$ patients. LND was strongly associated with prognostic factors and DSS. The modified N stage categories for penile SCC in our study incorporated LND and provided validated prognostic value with respect to the DSS of the penile SCC patients.

Patient survival is associated with the number of LNMs [13,14]. However, the number of LNMs removed is affected by various factors, such as the method of LN resection, the pathologist's assessment, and individual physiological variations [15,16]. LND is defined as the ratio of LNMs involved to the total number of LNs removed and thus incorporates both a surrogate of the extent of nodal dissection and the nodal disease burden in a single variable $[7,8]$. LND may accurately reflect heterogeneous patient characteristics and survival in other solid tumors $[4,11,17]$.

LND is also an effective predictor of penile disease burden. In a series of 73 penile cancer patients, Zhu et al. reported that LND correlated with the pelvic $L N M$ rate ${ }^{[18]}$. In one recent study, Lughezzani $G$ et al. demonstrated that LND was significantly associated with $\mathrm{N}$ stage, ENE, and bilateral LNM when stratified using a median value of $22 \%$ [9]. A similar finding was identified in our study. LND was associated with several prognostic factors. Patients with increased LNDs exhibited greater numbers of bilateral positive inguinal nodes, ENE, and pelvic LNM, as well as higher $\mathrm{T}$ and $\mathrm{N}$ stages. These data suggest that an increased LND corresponds to a worse survival rate. Moreover, $91.1 \%(51 / 56)$ of the ENEs, $94.7 \%$ $(54 / 57)$ of the bilateral LNMs, and 95.7\% (22/23) of the pelvic LNMs were characterized by LNDs $\geq 7.0 \%$. These findings indicate that the LND is effective in characterizing patients based on their distinct oncologic progressions. Further molecular analyses of a large population with penile cancer should be conducted to validate this hypothesis.

The LND outperforms number-based nodal staging in cancer prognostication. In 2009, Pettaway et al. proposed the prognostic value of LND in penile cancer based on an examination of 45 patients with $\mathrm{N}+$ penile cancer from the M. D. Anderson Cancer Center. The five-year DSS rates in the patients with LNDs $\leq 6.7 \%$ was $91.7 \%$, but this rate was only $23.3 \%$ among those with LNDs>6.7\% $(P<0.001)$ [7]. Similarly, Zhu et al. noted significant differences in survival rates according to LND [14]. Consistent with these studies, our data revealed that the three-year DSS rates were $90.9 \%, 66.5 \%$, and $22.2 \%$ in the different LND groups $\left(P<7.0 \% ; \quad 7.0-16.9 \%=0.006 ; \quad P_{7.0 \%-16.9 \%}\right.$; $\geq 17.0 \%=0.001$, Fig. $1 B$ ). Additionally, when included in a model with several factors, LND remained statisti- cally significant, whereas the other factors were no longer statistically significant. This finding is consistent with those reported for some cohorts $[7,9,14,19]$.

To our knowledge, our study represents the largest attempt to evaluate the value of LND in penile SCC patients with ILND. Additionally, we stratified the patients into LND tertiles and implemented a new proposal to evaluate the potential clinical usefulness of this metric. We found that LND classification yielded a positive predictive effect on survival. We presume that the addition of important information related to the status and extent of inguinal LNs could increase predictive accuracy. Table 5 provides data that support the hypothesis that the ND staging system increased the predictive accuracy in terms of DSS. The current treatment guidelines for penile cancer can be used to recommend individual treatments for patients $[1,2,18,20]$. Considering the heterogeneity that exists in such patients, the ND staging system could aid in the successful prediction of treatment outcomes with surgery alone. Moreover, ND staging could help to distinguish patients with advanced regional disease who should consider individualized therapy. Although the optimal cutoff of LND for penile SCC remains undetermined, this prognostic factor warrants further consideration. Given the small sample size and the fact that all of the results were used to generate the hypothesis, this new proposal should be considered exploratory.

We also recognize the limitations of this analysis. First, the optimal LND cutoff for penile SCC remains uncharacterized. The number of LNMs removed is affected by various factors. Irregular surgery, dynamic sentinel node biopsy, and lymph node biopsy could lead to false-negative results. Our study population was derived from one institution with few altered paradigms. All of the enrolled patients had an average of 24.0 nodes (range 8 to 63) and underwent bilateral ILND. We estimated the actual tumor loads for a few of the LNM patients based on the average number of harvested nodes. Second, adjuvant therapy (including adjuvant chemotherapy and/or radiotherapy) and pelvic lymphadenectomy may potentially affect other parameters. Pelvic lymphadenectomy was not routinely performed prior to 2009 because this unified standard was not recommended by the guidelines. Although we considered an adjuvant approach to lymphadenectomy, the results may still have been subject to the selection bias that was inherent in the design of this study. The predictive accuracies of each therapy should be assessed in external cohort populations to determine their validities for clinical prediction. Finally, data collection was performed retrospectively in this study. Therefore, the predictive accuracy of our results should be assessed 
in an external group to verify the clinical predictions.

In conclusion, LND is used as an instrument for stratifying patients based on their distinct oncologic progression. The ND system, which includes LND and the $7^{\text {th }}$ edition $\mathrm{N}$ classification, demonstrates an obvious positive effect on predictive accuracy and may aid in the selection of individual therapies for penile cancer patients.

\section{Acknowledgments}

The study is supported by grants from the Planned Science and Technology Project of Guangdong Province, China (2012B031800079), Sun Yat-sen University Cancer Center and State Key Laboratory of Oncology in Southern China, Guangzhou, China

\section{Authors' contribution}

Conception and design: Zai-Shang Li, Kai Yao, Peng Chen, Bin Wang, Qi-Wu Mi, Jie-Ping Chen, Yong-Hong Li, Chuang-Zhong Deng, Zhuo-Wei Liu, Zi-Ke Qin, Fang-Jian Zhou, Hui Han

Data acquisition: Zai-Shang Li, Kai Yao, Peng Chen, Bin Wang, Qi-Wu Mi , Jie-Ping Chen, Yong-Hong Li, Chuang-Zhong Deng, Zhuo-Wei Liu, Zi-Ke Qin

Data analysis and interpretation: Zai-Shang $\mathrm{Li}$,

Kai Yao, Fang-Jian Zhou, Hui Han

Drafting the manuscript: Zai-Shang Li, Kai Yao,

Critical revision of the manuscript for scientific and factual content: Statistical analysis: Fang-Jian Zhou, Hui Han

Supervision: Fang-Jian Zhou, Hui Han

(Both Zai-Shang Li and Kai Yao contributed equally to this work as a co-first author.)

\section{Competing Interests}

The authors have declared that no competing interest exists.

\section{References}

[1] Edge SB BDCM. AJCC Cancer Staging Manual, 7th ed. New York: Springer, 2009.

[2] O. W. Hakenberg ECSM, A. Necchi CPNW. Guidelines on Penile Cancer, EUA. 2014: 1-38,

[3] Clark PE. NCCN clinical practice guidelines in oncology (NCCN guidelines) penile cancer(2014). 2014.

[4] Fang AC, Ahmad AE, Whitson JM, et al. Effect of a minimum lymph node policy in radical cystectomy and pelvic lymphadenectomy on lymph node yields, lymph node positivity rates, lymph node density, and survivorship in patients with bladder cancer[J]. Cancer. 2010,116(8):1901-1908.

[5] Biffi $R$, Botteri E, Cenciarelli $S$, et al. Impact on survival of the number of lymph nodes removed in patients with node-negative gastric cancer submitted to extended lymph node dissection[J]. Eur J Surg Oncol. 2011,37(4):305-311.

[6] Pandey D, Mahajan V, Kannan RR. Prognostic factors in node-positive carcinoma of the penis[J]. J Surg Oncol. 2006,93(2):133-138.

[7] Svatek RS, Munsell M, Kincaid JM, et al. Association between lymph node density and disease specific survival in patients with penile cancer[J]. J Urol. 2009,182(6): 2721-2727.

[8] Li ZS, Yao K, Chen P, et al. Disease-specific survival after radical lymphadenectomy for penile cancer: Prediction by lymph node count and density[J]. Urol Oncol. 2014,32(6):893-900.
[9] Lughezzani G, Catanzaro M, Torelli T, et al. The relationship between lymph node ratio and Cancer-Specific survival in a contemporary series of patients with penile cancer and lymph node metastases[J]. Bju Int. 2013.

[10] Chang GJ, Rodriguez-Bigas MA, Skibber JM, et al. Lymph node evaluation and survival after curative resection of colon cancer: Systematic review[J]. J Natl Cancer Inst. 2007,99(6):433-441.

[11] Lee HH, Yoo HM, Song KY, et al. Risk of limited lymph node dissection in patients with clinically early gastric cancer: Indications of extended lymph node dissection for early gastric cancer[J]. Ann Surg Oncol. 2013,20(11):3534-3540.

[12] Yao K, Tu H, Li YH, et al. Modified technique of radical inguinal lymphadenectomy for penile carcinoma: Morbidity and outcome[J]. J Urol. 2010,184(2):546-552.

[13] Ravi R. Correlation between the extent of nodal involvement and survival following groin dissection for carcinoma of the penis[J]. Br J Urol. 1993,72(5 Pt 2):817-819.

[14] Zhu Y, Ye DW, Yao XD, et al. New N staging system of penile cancer provides a better reflection of prognosis[J]. J Urol. 2011,186(2):518-523.

[15] Johnson TV, Hsiao W, Delman KA, et al. Extensive inguinal lymphadenectomy improves overall 5-year survival in penile cancer patients: Results from the Surveillance, Epidemiology, and End Results program[J]. Cancer. 2010,116(12): 2960-2966.

[16] Zhu Y, Gu CY, Ye DW. Population-based assessment of the number of lymph nodes removed in the treatment of penile squamous cell carcinoma[J]. Urol Int. 2014,92(2):186-193.

[17] Solsona E, Iborra I, Rubio J, et al. Prospective validation of the association of local tumor stage and grade as a predictive factor for occult lymph node micrometastasis in patients with penile carcinoma and clinically negative inguinal lymph nodes[J]. J Urol. 2001,165(5):1506-1509.

[18] Zhu Y, Zhang SL, Ye DW, et al. Predicting pelvic lymph node metastases in penile cancer patients: A comparison of computed tomography, Cloquet's node, and disease burden of inguinal lymph nodes[J]. Onkologie. 2008,31(1-2):37-41.

[19] Zhu Y, Gu CY, Ye DW. Validation of the prognostic value of lymph node ratio in patients with penile squamous cell carcinoma: A population-based study[J]. Int Urol Nephrol. 2013,45(5):1263-1271.

[20] Pizzocaro G, Algaba F, Horenblas S, et al. EAU penile cancer guidelines 2009[J]. Eur Urol. 2010,57(6):1002-1012. 